\title{
Caracterización cromosómica de Hoplias malabaricus (Pisces, Erythrinidae) del Río Paraná, Argentina
}

\author{
Jorge, L.C. ${ }^{1}$; Moreira Filho, O. ${ }^{2}$ \\ ${ }^{1}$ Instituto de Ictiología, Facultad de Ciencias Veterinarias, UNNE, Sargento Cabral 2139, Corrientes (3400), \\ Argentina. Tel/fax 03783-425753. ${ }^{2}$ Departamento de Genética e Evolução, Univ. Federal de São Carlos, SP, \\ Brazil.E-mail: liliancj@vet.unne.edu.ar.
}

\begin{abstract}
Resumen
Jorge, L.C.; Moreira Filho, O.: Caracterización cromosómica de Hoplias malabaricus (Pisces, Erythrinidae) del Río Paraná, Argentina. Rev. vet. 21: 2, 132-135, 2010. Hoplias malabaricus presenta una amplia distribución geográfica, encontrándose en varias cuencas hidrográficas de algunos países de la región neotropical de América del Sur, donde asume valor económico. Dada su diversidad cariotípica, el objetivo del presente trabajo fue establecer la estructura cromosómica de esta especie en ejemplares provenientes del Río Riachuelo, afluente del Río Paraná en la localidad de Corrientes, Argentina. La población estudiada presentó un número diploide (2n) de 40 cromosomas del tipo meta-submetacéntricos, denominado "citotipo C" en estudios previos. No se evidenció ningún sistema de cromosomas sexuales. A través del bandeo $\mathrm{C}$ se localizó la heterocromatina constitutiva en posiciones pericentroméricas y teloméricas. Por medio de la coloración con nitrato de plata, en la región telomérica de seis cromosomas del complemento, se observaron marcaciones Ag-NOR (regiones organizadoras de nucléolos) positivas. Los resultados obtenidos proporcionan información adicional a los conocimientos preexistentes, corroborando la hipótesis que $\mathrm{H}$. malabaricus formaría parte de un complejo de especies.
\end{abstract}

Palabras clave: Hoplias malabaricus, cariotipo, bandeo C, Río Paraná, Argentina.

\begin{abstract}
Jorge, L.C.; Moreira Filho, O. Chromosome characterization of Hoplias malabaricus (Pisces, Erythrinidae) from Paraná River, Argentina. Rev. vet. 21: 2, 132-135, 2010. Hoplias malabaricus has a wide geographical distribution, being found in several river basins in neotropical regions of South American countries, where is considered a valuable resource. Due to its karyotype diversity, the aim of this study was to analyze the chromosomal structure of this species, from the Riachuelo River, tributary of the Paraná River in Corrientes, Northeastern Argentina. The population had a diploid number (2n) of 40 meta-submetacentric chromosomes, cited as "cytotype C" in previous research. There was no evidence of any type of sex chromosomes. $\mathrm{C}$ banding by constitutive heterochromatin was located in pericentromeric and telomeric position. By means of the silver nitrate staining it was observed $\mathrm{Ag}$ NOR positive bearings in the telomeric region of six chromosomes of the complement. The results provide additional information to the existing literature, corroborating the hypothesis that $H$. malabaricus is part of a complex of species.
\end{abstract}

Key words: Hoplias malabaricus, karyotype, C-banding, Paraná River, Argentina.

\section{INTRODUCCIÓN}

La familia Erythrinidae comprende los géneros Hoplias, Hoplerythrinus y Erythrinus. Hoplias malabaricus, especie conocida con el nombre vulgar de "tararira", es muy común en las cuencas de los ríos Paraná, Paraguay, de la Plata y Uruguay, así como en los ambientes leníticos dependientes de ellas ${ }^{12,22}$. Actualmente la especie está siendo seleccionada por piscicultores de Brasil debido a su abundancia en ríos y lagos, además de su aceptación por el mercado consumidor.

Recibido: 26 agosto 2010 / Aceptado: 4 octubre 2010
El estudio de la composición química del filet de tararira determinó que esta especie encuadra en la categoría "A", que incluye a los peces con valores extremadamente altos en proteínas $(>20 \%)^{23,25}$. Por otro lado, a través de la evaluación de los intervalos de gordura, $H$. malabaricus se clasifica entre los peces considerados como magros (hasta 2,5\% de lípidos), debido a que su contenido graso promedio es de $0,84 \%^{17,23}$.

Con relación a los estudios taxonómicos $H$. malabaricus es considerada como una única especie, sin embargo presenta patrones cariotípicos (citotipos) diferentes entre poblaciones aisladas en las diferentes cuencas hidrográficas de Brasil y Argentina, o entre pobla- 
ciones de distribución simpátrica cuyos ejemplares pueden ser colectados en sintopía. El cariotipo de estos ejemplares muestra variaciones numéricas $(2 \mathrm{n}=39$ a $2 \mathrm{n}=42$ ) y estructurales, observándose también diferentes sistemas de cromosomas sexuales $2,3,6-11,13,14,19,20,24$.

Debido a su diversidad cariotípica y a su interés para la piscicultura, el objetivo del presente trabajo fue analizar la estructura cariotípica de H. malabaricus proveniente del Río Riachuelo, afluente del Río Paraná en la Provincia de Corrientes, Argentina.

\section{MATERIAL Y MÉTODOS}

Se analizaron 25 especimenes de $H$. malabaricus, consiguiéndose resultados satisfactorio en 15 individuos ( 8 hembras y 7 machos), habiéndose registrado un total de 473 metafases. Antes del sacrificio se anestesió a los animales con metanosulfonato de tricaina MS222 (Finquel ${ }^{\circledR}$ ). Para el estudio de los cromosomas en tejidos somáticos, se empleó la técnica de "air drying" adaptada para peces. Los ejemplares fueron inyectados vía intraperitoneal con solución acuosa de colchicina $0,0125 \%$ en la proporción de $1 \mathrm{ml} / 100 \mathrm{~g}$ de peso del animal. Los especimenes permanecieron en peceras por aproximadamente 45 minutos.

La porción anterior del riñón se disoció en cubetas que contenían $10 \mathrm{ml}$ de solución hipotónica de $\mathrm{KCl}$ $(0,075 \mathrm{M})$. La suspensión celular resultante se mantuvo en estufa durante 30 minutos. Posteriormente el material se transfirió a un tubo de centrífuga, juntamente con algunas gotas de fijador (metanol:ácido acético, 3:1) y se centrifugó a $900 \mathrm{rpm}$ por 10 minutos. Se descartó el sobrenadante y las células se suspendieron en $10 \mathrm{ml}$ de fijador, centrifugándose 10 minutos a 900 rpm. Después

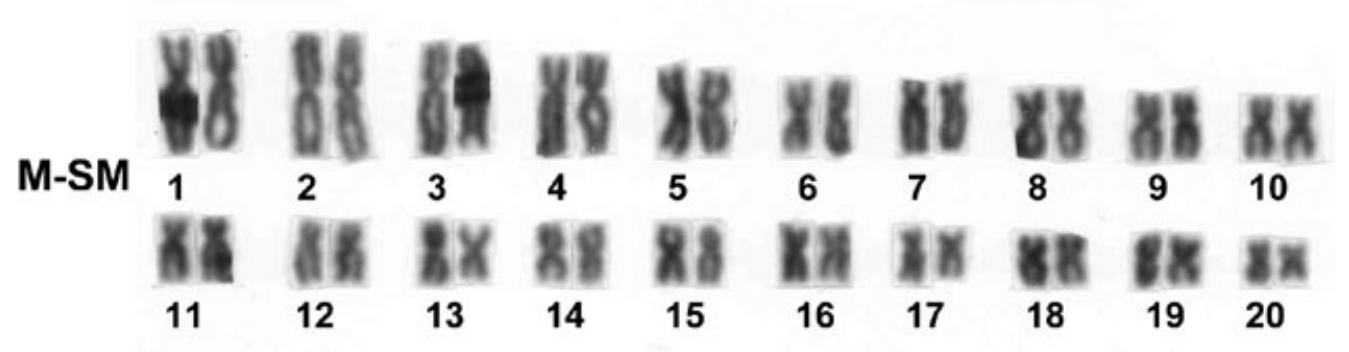

a
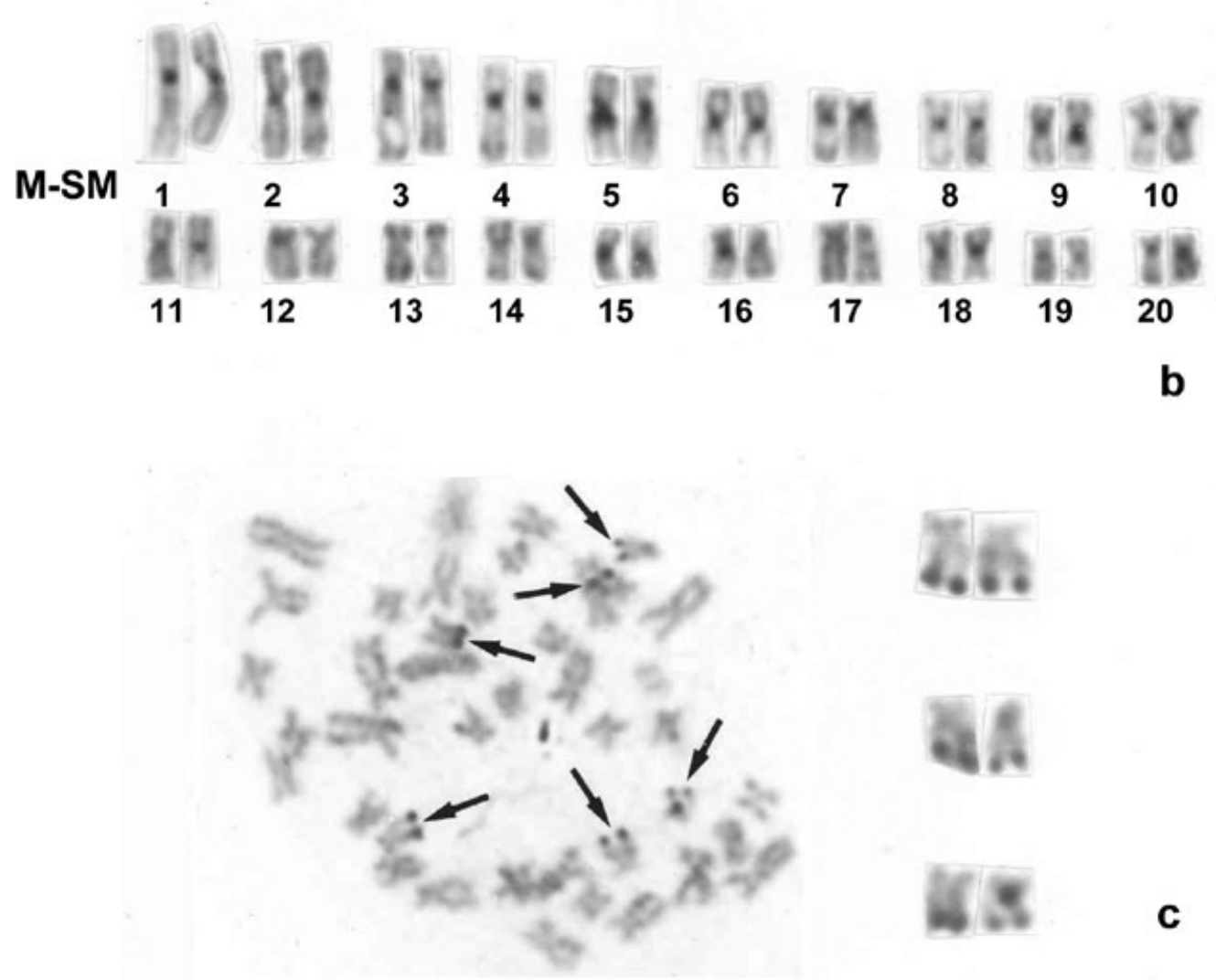

C

Figura 1. Cariotipo de Hoplias malabaricus coloración convencional de Giemsa (a), bandeo C (b) y coloración con nitrato de plata, NOR (c). 
de repetir nuevamente el procedimiento anterior las células fueron suspendidas en 1,5 ml de fijador y transferidas a un tubo Eppendorf. Para el análisis cromosómico, 3 gotas de suspensión celular fueron colocadas en un portaobjeto y coloreadas con Giemsa al 5\% diluido en tampón fosfato ( $\mathrm{pH} 6,8)$ durante 7 minutos ${ }^{1,21}$.

Por medio del bandeo $\mathrm{C}$ se evidenciaron los bloques de heterocromatina constitutiva; para la observación de bandas $\mathrm{C}+$ los portaobjetos con cromosomas se trataron con solución de $\mathrm{HCl}$ 0,2 $\mathrm{N}$ durante 10 minutos, a temperatura ambiente. Las preparaciones se lavaron en agua destilada e incubaron en solución de hidróxido de bario al $5 \%$ a $60^{\circ} \mathrm{C}$, por un período aproximado de 45 segundos. Posteriormente, los portaobjetos se lavaron rápidamente en $\mathrm{HCl} 0,2 \mathrm{~N}$ y agua destilada, incubándose luego en solución salina citratada doble concentración $(2 \mathrm{xSSC})$ durante 30 minutos a $60^{\circ} \mathrm{C}$. Después de un lavado final las preparaciones se colorearon con Giemsa al 5\% diluido en tampón fosfato $(\mathrm{pH} \mathrm{6,8)}$ durante 7 minutos ${ }^{26}$.

Para la identificación de las regiones organizadoras de nucléolos (NOR), se adicionaron dos gotas de solución acuosa de gelatina y cuatro gotas de solución acuosa de nitrato de plata $50 \%$ al portaobjeto con cromosomas, cubriéndose con un cubreobjeto. Esta preparación permaneció en estufa a $60^{\circ} \mathrm{C}$ por un período de aproximadamente 3 minutos, hasta alcanzar una coloración marrón dorado. Luego se retiró el cubreobjeto y el portaobjeto con el material a analizar se lavó con agua destilada y se coloreó durante 20 a 30 segundos en Giemsa al 5\% diluido en tampón fosfato $(\mathrm{pH} 6,8){ }^{16}$.

En el cariotipo se distribuyeron los pares homólogos en orden decreciente de tamaño. Los cromosomas se clasificaron como metacéntricos $(\mathrm{M})$ y submetacéntricos (SM) de acuerdo a su morfología y relación de brazos ${ }^{18}$.

\section{RESULTADOS Y DISCUSIÓN}

Investigaciones anteriores reportaron que $H$. malabaricus revela considerable diversidad cariotípica en varias poblaciones ${ }^{4}$. Sobre la base de sus rasgos macrocariotípico general, se identificaron en esta especie dos grandes grupos cromosómicos: uno compuesto por los citotipos A, B, C y D (grupo I) y otro por los citotipos E, F y G (grupo II) ${ }^{9}$.

Conforme a esta clasificación, los ejemplares de $H$. malabaricus estudiados en el presente trabajo formarían parte del citotipo $\mathrm{C}$ (grupo I), caracterizado por presentar un número diploide (2n) igual a 40 cromosomas en ambos sexos, con un cariotipo constituido por 15 pares $\mathrm{M}$ y 5 pares SM, todos ellos identificados por mediciones cromosómicas, con un número fundamental (NF) igual a 80 (Figura 1a). En los individuos analizados no se observó ningún sistema de cromosomas sexuales, siendo éstos identificados en algunas poblaciones de $H$. malabaricus de Brasil ${ }^{3,8-11,13}$.

La distribución de la heterocromatina constitutiva (Figura 1b) en los cromosomas que se sometieron a la técnica de bandeo-C puede ser así resumida: (a) bloques pericentroméricos en todos los cromosomas del cariotipo, siendo que en el par número cinco se evidenció una banda pericentromérica grande, presente en el brazo largo; y (b) bloques teloméricos en los brazos largos de varios M y SM (cromosomas 13, 14 y 20 M; 3, 4 y 7 SM). Se observaron también bloques teloméricos en el brazo corto del par $13 \mathrm{M}$.

En otros trabajos, la heterocromatina constitutiva mostró una distribución patrón en todas las poblaciones analizadas de $H$. malabaricus $6,7,10,11,13,14,19$, siendo pericentromérica en todos los cromosomas del complemento y telomérica en algunos cromosomas; esta última condición fue semejante a la hallada en la población aquí estudiada.

El análisis de las regiones organizadoras de nucléolos coloreadas con nitrato de plata (Ag-NORs) reveló un número variable de marcaciones. En esta población se observó un número de 4 a 6 cromosomas con regiones $\mathrm{Ag}-\mathrm{NORs}+$, siendo cuatro el número más frecuente. Las marcaciones se localizaron en la región telomérica del brazo largo de cromosomas del tipo meta/ submetacéntricos (Figura 1c).

De manera similar a lo encontrado en otras poblaciones de Argentina y Brasil, ejemplares de H. malabaricus del Río Riachuelo presentaron NORs múltiples, ubicadas en posición telomérica. Se admite que la familia Erythrinidae está caracterizada por poseer NORs múltiples, siendo ésta una condición común en este grupo de peces, con excepción de $H$. lacerdae. La NOR puede presentarse en posición telomérica ( $H$. malabaricus y Erythrinus), intersticial (H. lacerdae) y telomérica e intersticial (Hoplerythrinus) ${ }^{5,13-15,19,24}$.

Se concluye que los resultados citogenéticos de este trabajo, juntamente con los datos existentes en la bibliografía, resaltan la necesidad de una revisión taxonómica en $H$. malabaricus debido a que especies distintas pueden estar representadas entre los citotipos detectados.

\section{REFERENCIAS}

1. Bertollo LA, Takahashi CS, Moreira-Filho O. 1978. Cytotaxonomic considerations on Hoplias lacerdae (Pisces, Erythrinidae). Brazil J Genet 2: 103-120.

2. Bertollo LA, Takahashi CS, Moreira-Filho O. 1979. Karyotypic studies of two allopatric populations of the genus Hoplias (Pisces, Erythrinidae). Brazil J Genet 2: 17-37.

3. Bertollo LA, Takahashi CS, Moreira-Filho O. 1983. Multiple sex chromosomes in the genus Hoplias (Pisces, Erythrinidae). Cytologia 48: 1-12.

4. Bertollo LA, Moreira-Filho O, Galetti PM. 1986. Cytogenetics and taxonomy: considerations based on chromosome studies of freshwater fish. J Fish Biol 28: 153-159.

5. Bertollo LA. 1996. The nucleolar organizer regions of Erythrinidae fish. An uncommon situation in the genus Hoplias. Cytologia 61: 75-81. 
6. Bertollo LA, Moreira-Filho O, Fontes MS. 1997. Karyotipic diversity and distribution in Hoplias malabaricus (Pisces, Erythrinidae): cytotipes with $2 \mathrm{n}=40$ chromosomes. Brazil J Genet 20: 237-242.

7. Bertollo LA, Fontes MS, Fenocchio AS, Cano J. 1997. The $\mathrm{X}_{1} \mathrm{X}_{2} \mathrm{Y}$ sex chromosome system in the fish Hoplias malabaricus. I. G-, C- and chromosome replication banding. Chromosome Res 5: 493-499.

8. Bertollo LA, Mestriner CA. 1998. The $\mathrm{X}_{1} \mathrm{X}_{2} \mathrm{Y}$ sex chromosome system in the fish Hoplias malabaricus (Pisces, Erythrinidae). II. Meiotic analyses. Chromosome Res 6: 141-147.

9. Bertollo LA, Born GG, Dergam JA, Fenocchio AS, Moreira-Filho O. 2000. A biodiversity approach in the neotropical Erythrinidae fish, Hoplias malabaricus. Karyotypic survey, geographic distribution of cytotypes and cytotaxonomic considerations. Chromosome Res 8: 603-613.

10. Born GG, Bertollo LA. 2000. An XX/XY sex chromosome system in a fish species, Hoplias malabaricus, with a polymorphic NOR-bearing X chromosome. Chromosome Res 8: 111-118.

11. Born GG, Bertollo LA. 2006. A new sympatric region for distinct karyotypic forms of Hoplias malabaricus (Pisces, Erythrinidae). Braz J Biol 66: 205-210.

12. Casciotta J, Almirón A, Bechara J. 2005. Peces del Iberá. Hábitat y diversidad, Ed. Graficar, La Plata (Argentina), p. 154-155.

13. Dergam JA, Bertollo LA. 1990. Karyotypic diversification in Hoplias malabaricus (Osteichthyes, Erythrinidae) of the São Francisco and Alto Paraná basins, Brazil. Brazil J Genet 13: 755-766.

14. Ferreira RH, Fonseca CG, Bertollo LA, Foresti F. 1989. Cytogenetics of fishes from Parque Forestal do Rio Doce (MG). I. Preliminary study of "Hoplias malabaricus" (Pisces, Erythrinidae) from Lagoa Carioca and Lagoa dos Patos. Brazil J Genet 12: 219-226.

15. Giuliano LA. 1986. Estudos citogenéticos em Hoplerythrinus unitaeniatus (Pisces, Erythrinidae) de diferentes bacias hidrográficas brasileiras. Tesis de Maestria, Universidade Federal de São Carlos, SP, Brasil, p. 17-54.
16. Howell WM, Black DA. 1980. Controled silver-staining of nucleolus organizer regions with a protective colloidal developer: a 1-step method. Experientia 36: 1014-1015.

17. Jacquot, R. 1961. Organic constituents of fish and foods. In: Fish and food (Borgsrom G Ed), Academic Press, New York, p. 144-192.

18. Levan A, Fredga K, Sandberg AA. 1964. Nomenclature for centromeric position on chromosomes. Hereditas 52: 201-220.

19. Lopez PA, Fenocchio AS. 1994. Confirmation of two different cytotypes for the neotropical fish Hoplias malabaricus Gill 1903 (Characiformes). Cytobios 80: 217-221.

20. Lopes PA, Alberdi AJ, Dergam JA, Fenocchio AS. 1998. Cytotaxonomy of Hoplias malabaricus (Osteichthyes, Erythrinidae) in the Aguapey river (Province of Corrientes, Argentina). Copeia 2: 485-487.

21. Moreira-Filho O, Bertollo LA. 1991. Extraction and use of the cephalic kidney for chromosome studies in small fish. Brazil J Genet 14: 1085-1090.

22. Ringuelet RA, Aramburu RH, Aramburu AA. 1967. Los peixes argentinos de agua dulce, Ed. Comisión de Investigaciones Científicas (CIC), La Plata (Argentina), p. 226-229.

23. Santos AB, Melo JF, Lopes PR, Malgarim MB. 2000. Chemical composition and filet yield in Traíra (Hoplias malabaricus). Rev FZVA (Uruguaiana) 7/8: 140-150.

24. Scavone MD, Bertollo LA, Cavallini MM. 1994. Sympatric occurrence of two karyotypic forms of Hoplias malabaricus (Pisces, Erythrinidae). Cytobios 80: 223-227.

25. Stansby ME. 1962. Proximate composition of fish. In: Fish in nutrition (E. Heen, R. Kreuzer Ed), Fishing News Books Ltd, London, p. 1-59.

26. Sumner AT. 1972. A simple technique for demonstrating centromeric heterochromatin. Exp Cell Res 75: 304-306. 